\title{
A equidade intergeracional e a taxa de desconto em impactos ambientais de longo prazo
}

Intergerational equity and discount rate in long term environmental impacts

\author{
Ilana Dalva Ferreira (1) \\ Denise Imbroisi (2) \\ (1) Confederação Nacional da Indústria \\ (2) Universidade de Brasília
}

\begin{abstract}
This paper evaluates the concept of intergenerational equity in the environmental evaluation of projects with long term impacts in relation to the determination of the discount rate. The concepts of intergenerational equity in the Economy and the ways found to include this aspect in the definition of the discount rate used in the economic measurement of the environmental costs with long term impacts are analyzed. The proposals presented by Padilla (2002) and the application of the Ramsey Formula with equivalent safety are adopted in the analysis. The analyzes conducted showed that there is a significant difference in the adoption of the discount rate, which is said to be sustainable compared to the market rate in the Brazilian case. Some suggestions in terms of decision making are presented at the end.
\end{abstract}

\section{Resumo}

Este trabalho avalia o conceito de equidade in-

\section{Keywords}

sustainable development; discount rate; intergenerational equity.

JEL Codes Q40. tergeracional na avaliação ambiental de projetos com impactos no longo prazo no que tange à determinação da taxa de desconto. São analisados os conceitos de equidade intergeracional na Economia e as formas encontradas para incluir esse aspecto na definição da taxa de desconto utilizada na mensuração econômica dos custos ambientais com impactos no longo prazo. As propostas apresentadas por Padilla (2002) e a aplicação da Fórmula de Ramsey com segurança equivalente são adotadas na análise. As análises conduzidas apontaram que há uma diferença significativa na adoção da taxa de desconto dita sustentável em comparação com a de mercado no caso brasileiro. Algumas sugestões em termos de tomada de decisão são apresentadas no final.

\section{Palavras-chave}

desenvolvimento sustentável; equidade intergeracional; taxa de desconto.

Códigos JEL Q40. 


\section{Introdução}

O trade-off entre gerar desenvolvimento econômico no presente ou preservar os recursos naturais para as gerações futuras é um dos grandes dilemas na busca pelo desenvolvimento sustentável. $O$ processo produtivo interfere nos processos naturais e a maioria dos problemas ambientais estaria associada ao consumo de recursos naturais e despejo de resíduos ao meio ambiente. A preocupação com as gerações futuras é pertinente já que as interações ecológicas não respeitam as fronteiras dos indivíduos ou governos, e nem as geracionais. Em outras palavras, as externalidades são dinâmicas e intertemporais (BERGH, 2010). Portanto, a depleção dos recursos naturais para a concretização dos processos produtivos que saciam as nossas necessidades pode gerar limitações para a satisfação das necessidades das gerações futuras.

A geração de energia elétrica, por exemplo, tem impactos ambientais potenciais relevantes. O planejamento de uma matriz de geração de energia elétrica envolve também a escolha de quais impactos ambientais podem ser aceitos, controlados e compensados. A atual preocupação com as mudanças climáticas tem levado os governos a se preocuparem em planejar uma matriz energética com menor emissão de gases de efeito estufa. Conforme projeções do UK Energy Research Center (2013), a matriz elétrica seria largamente "descarbonizada" em 2030 com uma redução de $90 \%$ no nível de emissão de 2010. A intensidade cairia à zero (isto é, "descarbonização" completa) a partir de 2035 por conta da enorme implantação de energia nuclear pós-2025. De acordo com Mez (2012), há atualmente 63 reatores nucleares em construção e 156 usinas nucleares em planejamento no mundo.

Contudo, a busca pela redução da emissão dos gases de efeito estufa pode gerar outro impacto que apresenta grande dificuldade de gerenciamento: os rejeitos nucleares. A disposição final de rejeitos nucleares ainda não foi equacionada e envolve impactos potenciais para diversas gerações futuras. O problema é que não há clareza de quais são os reais impactos potenciais e tampouco há definição de quais instrumentos econômicos devem ser adotados para avaliar impactos no longo prazo.

Este trabalho tem como objetivo avaliar o conceito de equidade intergeracional na avaliação ambiental de projetos com impactos no longo prazo no que tange à determinação da taxa de desconto. 
Na primeira seção é apresentada uma revisão teórica sobre o conceito de equidade intergeracional na economia com a análise de qual abordagem seria adequada para o caso dos projetos com impactos ambientais no longo prazo. A segunda seção descreve diversas formas do cálculo da taxa de desconto aplicada em análises de longo prazo com ênfase na equidade intergeracional. A quarta seção exemplifica as diferenças de abordagem para o setor elétrico e os comentários finais estão descritos na última seção.

\section{A equidade intergeracional e a teoria econômica}

A teoria econômica prevê que é muito comum o trade-off entre equidade e eficiência. Okun (1975) afirmou que é impossível evitar o conflito entre equidade e eficiência no conceito econômico. $O$ autor mostra que no processo de se promover equidade, haveria o efeito de vazamento (leaking bucket) em que no processo de realocação de recursos de um indivíduo rico para o pobre, haveria a redução desse recurso em termos de benefícios para o indivíduo com menor renda. Esse vazamento ocorreria por conta dos custos administrativos da política de equidade, alterações na disposição a poupar e investir dos indivíduos, dentre outros fatores. Consequentemente, a tentativa de se atingir a equidade, geraria uma alocação ineficiente dos recursos.

Contudo, uma série de trabalhos mostram que é possível compatibilizar tais aspectos em situações específicas. Thurow (1981), por exemplo contribuiu significativamente para o debate das relações entre a equidade, eficiência, justiça social e redistribuição. $\bigcirc$ autor mostra que a relutância em prosseguir ou o desejo de recuar são muitas vezes justificadas como uma reação apropriada ao trade-off áspero entre equidade e eficiência e desmistifica a ideia de que mais igualdade significa menos crescimento. O autor mostra, com base nos dados de diversos países, que não há correlação entre desempenho, o grau de igualdade ou a extensão do esforço de redistribuição de renda.

$\mathrm{Na}$ área ambiental, Pascual et al. (2010) abordam a relação entre eficiência e equidade nos pagamentos de serviços ambientais. Os autores afirmam que diferentes tipos de fatores institucionais determinam que haja combinações de equidade-eficiência e que podem ser potencialmente viáveis. 
Tal discussão é especificamente interessante quando se busca analisar formas de desconto que considerem as gerações futuras e, assim, permitam uma análise sustentável dos projetos.

A análise da equidade intergeracional dentro da teoria econômica precisa considerar dois conceitos distintos que guiam os pressupostos dos autores: a sustentabilidade fraca e a sustentabilidade forte.

Tais conceitos referem-se ao papel e à relação do capital natural com os outros tipos de capital. Quando o capital natural é indefinidamente substituível por outros tipos de capital tem-se a visão denominada sustentabilidade fraca. Essa análise é caracterizada por um maior otimismo da substituição do capital natural pelo capital de produção humana (MUELLER, 2007). Essa substituição instantaneamente considera que a busca pelo crescimento e desenvolvimento econômico já incluiria a equidade intergeracional, pois o capital físico oriundo do desenvolvimento tecnológico substituiria o capital natural e não afetaria as condições das gerações futuras.

Já a sustentabilidade forte defende que a substitutabilidade entre o capital natural e os outros tipos de capital é limitada. A substituição dos capitais é possível até certo ponto, contudo, a relação de complementaridade prevalece quando há escassez relativa do capital natural.

A maior parte dos autores analisados apresentam uma visão otimista quanto à capacidade de substituição do capital natural por outros tipos de capital, como será exposto a seguir.

\subsection{Principais visões da equidade intergeracional na Economia na década de 1970 e 1980.}

$\mathrm{Na}$ economia, Solow (1974) discutiu a questão da equidade em formato de crítica ao trabalho de Rawls (1971). Rawls (1971) afirma que a inequidade na distribuição de bem-estar e utilidade só seria justificada se fosse apresentada como condição a melhora da utilidade dos mais pobres. Essa análise seria uma aplicação filosófica do Princípio Max-Min. Esse princípio busca minimizar o custo de um cenário pior (custo maximizado). Pode ainda ser interpretado como a maximização de um ganho já minimizado. Assim, Solow (1974) explorou as consequências da aplicação direta do Princípio Max-Min no problema de acumulação ótima de capital entre as gerações. 
O trabalho de Solow (1974) é relevante por ser uma tentativa primária de modelagem econômica do conceito de equidade intergeracional. Dentre as conclusões, foi observado que a única maneira de manter o bem-estar social ao longo das gerações seria com a manutenção do estoque de capital de modo a acompanhar o crescimento populacional. Caso a geração presente consuma mais de forma a reduzir o estoque de capital, a geração futura teria uma situação pior. $O$ modelo também considera os recursos naturais exauríveis no processo de análise, porém, esse componente se torna indiferente quando o desenvolvimento tecnológico é incluído na análise.

Para Solow (1974), a elasticidade de substituição entre o capital natural e outros tipos de capital deve ser no mínimo unitária. Ou seja, o capital acumulado seria suficiente para compensar a depleção dos recursos naturais. A visão de Solow (1974) é similar à de outros trabalhos da época como The Optimal Depletion of Exhaustible Resources (Dasgupta e Heal, 1974) e Growth with Exhaustible Natural Resources: Efficient and Optimal Growth Paths (Stiglitz, 1974).

Uma das principais conclusões da análise de Dasgupta e Heal (1974) é que a maximização da utilidade estaria associada à maximização do Valor Presente Líquido (VPL) por meio de uma taxa de desconto. O interessante é que o trabalho conclui que o resultado do VPL é sombrio para as gerações futuras. Após o alcance de um pico inicial, o consumo e a utilidade eventualmente chegarão a zero no longo prazo. Esse resultado é uma consequência direta de uma taxa de desconto positiva combinada com a escassez inerente da fonte não renovável. Esse processo claramente não é compatível com o conceito de desenvolvimento sustentável.

Para Stiglitz (1974), uma maneira de evitar esse resultado indesejável seria por meio do progresso tecnológico. Em seu modelo, a taxa exógena do progresso técnico é dada como suficientemente grande para compensar os efeitos do esgotamento de recursos. Este pressuposto implica que o caminho para o VPL ótimo pode ter aumento sustentado do consumo per capita (mesmo com uma população crescente, o que é omitido no modelo de Dasgupta e Heal (1974).

Nos três modelos, os recursos naturais são finitos, não renováveis e essenciais para a produção, não sendo completamente ignorados, como tinha ocorrido em grande parte da teoria de crescimento econômico. No entanto, o capital natural é indefinidamente substituível por outros tipos 
de capital por meio de uma função Cobb-Douglas. Essa visão de substitutabilidade é claramente a de sustentabilidade fraca.

O trabalho de Dasgupta e Mitra (1983) mostra uma pequena diferença em relação aos três anteriores. Eles buscam averiguar se a equidade intergeracional é compatível com a alocação eficiente de recursos exauríveis. Para isso, é necessário encontrar um patamar em que o consumo das gerações futuros fosse positivo. Os autores pressupõem que a condição necessária e suficiente para que o consumo das gerações futuras seja positivo é a participação do capital no produto superior à participação dos recursos exauríveis no produto. Assim, existiria um nível de produto eficiente e equânime. A grande contribuição do trabalho está na análise de que o investimento não pode ser superior à capacidade de recuperação dos recursos exauríveis em um patamar de equidade eficiente.

\subsection{Principais visões da equidade intergeracional na Economia na década de 1990.}

Outros autores com trabalhos relevantes sobre equidade entre as gerações são Howarth e Norgaard (1990). Os autores mostram que os resultados da teoria neoclássica do bem-estar no que diz respeito à dotação inicial de equidade e eficiência podem ser traduzidos em um modelo estático para um contexto intergeracional. Diferentes dotações de recursos para duas gerações resultaram em diferentes distribuições de riqueza, todas elas eficientes, mas, obviamente, com implicações na equidade. Howarth e Norgaard apresentam um modelo estendido em 1992 e a principal conclusão é que o caminho do consumo através do tempo e da valoração marginal da externalidade ambiental, baseada na taxa eficiente de poluição, depende da distribuição da riqueza entre as gerações.

Assim, mesmo em teoria, não há nenhuma noção clara de como valorar um custo ambiental de forma 'correta' entre as gerações. O valor varia de acordo com a mudança da visão da sociedade sobre o futuro e a expressa por uma taxa de desconto ou algum outro critério de sustentabilidade.

Em 1997, Page compara duas abordagens para resolver o problema de alcançar os objetivos socialmente escolhidos de eficiência e equidade intergeracional. Na primeira, é realizada uma análise custo-benefício padrão e posteriormente considera-se o aspecto da equidade entre as gerações de 
forma separada. Na segunda abordagem, o aspecto da equidade é considerado desde o começo. Page (1997) define a equidade intergeracional como a manutenção do capital total (naturais e físicos) ao longo do tempo. $\mathrm{O}$ autor discute as dificuldades de se manter toda a base de recursos por conta dos limites da substitutabilidade entre os tipos de capital por meio do progresso técnico. $O$ trabalho aponta que o preceito da sustentabilidade envolve adotar ações tendo como base a manutenção dos recursos naturais. Essa abordagem se aproxima da sustentabilidade ambiental forte em que a substitutabilidade entre os tipos de capital é limitada conforme a classificação já exposta por Mueller (2007).

\subsection{Principais visões da equidade intergeracional na Economia pós anos 2000.}

O trabalho de Asheim, Buchholz e Tungodden (2001) discute como os modelos que avaliam a equidade intergeracional para infinitas gerações podem não apresentar soluções economicamente ótimas. $O$ estudo mostra que um axioma da equidade de tratamento, combinado com o axioma de Pareto (eficiência) seria suficiente para descartar caminhos não sustentáveis de utilidade intergeracionais como soluções ótimas. Esse resultado depende apenas do pressuposto de que a utilidade de qualquer geração pode ser mensurada, ordenada e comparada com os níveis de utilidade de outras gerações. Por fim, é demonstrado que as preferências sociais são eficientes e equitativas sob a condição de que a tecnologia gera aumento da produtividade. Os autores concluem que a produtividade seria suficiente para garantir a existência de preferências sociais completas com eficiência e equidade, resultando em uma única e sustentável solução para o problema da justiça intergeracional.

De forma mais completa, o trabalho de Padilla (2002) averigua as limitações da análise econômica sobre as questões intergeracionais e analisa as alternativas sugeridas na literatura. $O$ autor esclarece que uma consideração adequada das gerações futuras pressupõe que a análise custo-benefício deve estimar que tais impactos (positivos e negativos) serão sentidos por diferentes gerações. Além disso, seria preciso considerar que as gerações futuras têm direito a um ambiente econômico e ecológico não deteriorado. 
Padilla (2002) ressalta que é primordial superar as limitações da análise econômica convencional em relação ao desenvolvimento sustentável, como por exemplo, no caso de externalidades intergeracionais. Os problemas intergeracionais são oriundos do fato de que as ações atuais determinam a capacidade econômica e ecológica que as gerações futuras usufruirão. Há externalidades porque os custos das decisões da geração atual que impactarão as vidas das gerações futuras não são devidamente contabilizados e podem causar efeitos irreversíveis.

A análise convencional considera que a externalidade é uma falha de mercado. Nesse caso específico, não há mercado e as soluções convencionais não são válidas. Assim, o Teorema de Coase não pode ser aplicado, porque não existe a possibilidade das partes envolvidas entrarem em um acordo já que as gerações futuras não estarão presentes ou poderão ser representadas.

No caso de uma solução Pigouviana, a valoração futura do mercado seria hipotética também por conta da não participação das gerações futuras, então o valor dado ao futuro seria arbitrário. Além disso, a internalização das externalidades seria administrada por uma instituição em que não há representatividade das gerações futuras. Nesse caso, o questionamento pertinente estaria na legitimidade da análise convencional onde o futuro é avaliado como algo contingente nas preferências atuais (PADILLA, 2002).

O próprio conceito convencional de alocação eficiente dos recursos não inclui o aspecto intergeracional. Isso porque as análises se referem a mercados baseados nas preferências atuais, assim como o teste de compensação Kaldor-Hicks que afirma que se os benefícios forem superiores aos custos, seria eficiente adotar tal projeto sem considerar aspectos distributivos. Tal critério perde sua legitimidade na análise intergeracional. Deve-se questionar o direito das gerações atuais de impor impactos ambientais às futuras gerações. Na falta de compensações efetivas, essa parece ser uma contestação cabível. Tal análise acaba tornando necessária a inclusão de um viés moral na definição das dotações dos recursos e as possíveis distribuições. $O$ autor expõe duas regras orientadoras no processo decisório.

A primeira seria a Regra da Inalienabilidade que implica a não alteração nos direitos das gerações futuras. A segunda seria a Regra da Compensação, que prevê ações atuais com o intuito de retomar a dotação original de 
direitos das gerações futuras. A primeira impediria qualquer alteração e a segunda permitiria uma forma de compensação. A Regra da Compensação permitiria amenizar as ineficiências da primeira. Porém, como ela prevê certa substitutabilidade entre os tipos de capital, a sua aplicação tornaria necessária uma maior avaliação dos impactos globais da ação avaliada.

Já Chichilnisky (2009) trouxe o aspecto comportamental para a real implementação do desenvolvimento sustentável e, consequentemente, da equidade intergeracional na economia. A autora afirma que o desafio estaria em como definir valores econômicos que vão além das necessidades individuais e atuais identificadas pelo mercado e incluir as necessidades das futuras gerações. Nesse sentido, uma mudança nas preferências da população seria primordial e viria da consciência sobre as reais e novas restrições físicas de longo prazo dos recursos naturais como, por exemplo, a extinção de alguma espécie.

Em um trabalho de 2012, Chichilnisky avalia como as premissas do desenvolvimento sustentável, como a equidade intergeracional, vão de encontro aos objetivos do mercado que normalmente focam no curto prazo. O trabalho é desenvolvido com base no conceito de impaciência e com a ideia de que um dólar hoje vale mais do que um dólar amanhã, o que já demonstra um viés da economia pelo presente.

De acordo com Chichilnisky (2012), é possível reverter o viés pelo presente da economia definindo novos tipos de mercado com comerciantes que apresentam preferências sustentáveis. Como o mercado segue o comportamento dos comerciantes, quando eles têm preferências sustentáveis, o mercado se torna sustentável. A nova estrutura de preferências vai além do axioma da impaciência, ela tem como base os axiomas que requerem um tratamento equitativo entre a geração atual e as futuras. Mercados com preferências sustentáveis superam o axioma da impaciência, porque eles não são nem ditatoriais para o presente, nem para o futuro.

O Quadro 1 resume o posicionamento dos autores citados. Apesar da relevância dos trabalhos apresentados, o estudo de Padilla (2002) apresenta uma visão mais aplicável de equidade intergeracional por definir ações a serem adotadas no processo decisório de execução de projetos tendo como base os impactos ambientais averiguados. Como um dos objetivos deste artigo é sugerir algumas condutas no processo decisório de empreendimentos com impactos de longo prazo, o trabalho de Padilla (2002) é o que melhor se adequa às complexidades deste processo. 
Quadro 1 Resumo dos autores que tratam de Equidade Intergeracional

\begin{tabular}{|c|c|c|}
\hline Autor & $\begin{array}{l}\text { Visão de Sustentabilidade e } \\
\text { de Equidade Intergeracional }\end{array}$ & $\begin{array}{l}\text { Apresenta } \\
\text { proposta } \\
\text { de análise } \\
\text { de projetos }\end{array}$ \\
\hline $\begin{array}{l}\text { Solow } \\
(1974)\end{array}$ & $\begin{array}{l}\text { Sustentabilidade Fraca. } 0 \text { desenvolvimento tecnológico seria } \\
\text { suficiente para atender a necessidade das gerações futuras. }\end{array}$ & Não \\
\hline $\begin{array}{l}\text { Dasgupta e Heal } \\
\text { (1974) }\end{array}$ & $\begin{array}{l}\text { Sustentabilidade Fraca. } 0 \text { desenvolvimento tecnológico seria } \\
\text { suficiente para atender a necessidade das gerações futuras. }\end{array}$ & Não \\
\hline $\begin{array}{l}\text { Stiglitz } \\
(1974)\end{array}$ & $\begin{array}{l}\text { Sustentabilidade Fraca. } 0 \text { desenvolvimento tecnológico seria } \\
\text { suficiente para atender a necessidade das gerações futuras. }\end{array}$ & Não \\
\hline $\begin{array}{l}\text { Dasgupta e Mitra } \\
\text { (1983) }\end{array}$ & $\begin{array}{l}\text { Sustentabilidade Fraca com reconhecimento da importância } \\
\text { da limitação da extração dos recursos exauríveis. }\end{array}$ & Não \\
\hline $\begin{array}{l}\text { Howarth e Norgaard } \\
(1990,1992)\end{array}$ & $\begin{array}{l}\text { Sustentabilidade Fraca. A utilização de uma taxa de descon- } \\
\text { to seria suficiente para representar a visão das gerações } \\
\text { futuras. }\end{array}$ & Não \\
\hline $\begin{array}{l}\text { Page } \\
(1997)\end{array}$ & $\begin{array}{l}\text { Sustentabilidade Forte. A equidade intergeracional seria } \\
\text { atendida com a manutenção do capital total (naturais e } \\
\text { físicos). Contudo, questiona os limites da substitutabilidade. }\end{array}$ & Não \\
\hline $\begin{array}{l}\text { Asheim, Buchholz, } \\
\text { Tungodden } \\
(2001)\end{array}$ & $\begin{array}{l}\text { Sustentabilidade Fraca. As preferências sociais são eficien- } \\
\text { tes e equânimes sob a condição de que a tecnologia gera } \\
\text { aumento da produtividade. }\end{array}$ & Não \\
\hline $\begin{array}{l}\text { Padilla } \\
(2002)\end{array}$ & $\begin{array}{l}\text { Considera a sustentabilidade fraca para impactos em peque- } \\
\text { nas escalas, locais e regionais, e a sustentabilidade forte } \\
\text { para impactos no sistema ecológico global. }\end{array}$ & Sim \\
\hline $\begin{array}{l}\text { Chichilnisky } \\
(2009,2012)\end{array}$ & $\begin{array}{l}\text { Sustentabilidade fraca. Foca na mudança comportamental } \\
\text { como forma de aumentar a relevância dos impactos ambien- } \\
\text { tais nos mercados. }\end{array}$ & Não \\
\hline
\end{tabular}

Fonte: Elaboração própria com base nas referências mencionadas.

\section{Taxa de desconto e equidade intergeracional}

A análise econômica de projetos é um importante instrumento na tomada de decisão de gestores públicos. A escassez de recursos torna necessária a priorização de ações como escolha de política, programa ou projeto mais adequado para sanar ou amenizar problemas da sociedade. Porém, esse processo de escolha não é trivial. A adoção de políticas e programas, assim como a execução de projetos, gera múltiplos impactos na sociedade. 
A definição da ação a ser tomada precisa considerar impactos diversos como os financeiros, sociais e ambientais.

A mensuração econômica dos impactos positivos e negativos (Análise Custo-Benefício - ACB) é um instrumento prático para averiguar quão desejável um projeto é por meio da enumeração e quantificação dos custos e benefícios envolvidos na decisão de realizar uma política ou empreendimento. A mensuração econômica dos impactos também desempenha um papel importante de subsídio ao processo de tomada de decisão.

Apesar de intuitivo, o processo não deixa de ser complexo exatamente por expandir os critérios na tomada de decisão para além do financeiro. Um projeto, por exemplo, pode apresentar elevado retorno financeiro, mas impactos sociais destrutivos. A análise puramente financeira considera apenas o retorno monetário do empreendimento sem avaliar as externalidades causadas por tal ação. Conforme exposto por Contador (2000), a análise econômica mensura o preço econômico, muitas vezes divergente do preço de mercado e busca exatamente averiguar os efeitos diretos e indiretos que podem ser causados à sociedade.

A ausência de um preço de mercado ou a sua divergência em relação ao preço social torna necessária a valoração econômica. De acordo com a Associação Brasileira de Normas Técnicas - ABNT (2008), valoração ambiental é a identificação do valor de um recurso ambiental ou do custo de reparação de um dano ambiental. Seroa da Motta (1997) em seu "Manual de Valoração Econômica do Meio Ambiente" decompõe o que seria o Valor Econômico de um Recursos Ambiental (VERA), composto pelo:

- Valor de uso direto (VUD): valor associado à utilização atual de um recurso ambiental.

- Valor de uso indireto (VUI): valor associado ao benefício atual das funções ecossistêmicas do recurso.

- Valor de opção (VO): valor associado ao valor de uso direto e indireto que só serão usufruídos no futuro (benefício potencial).

- Valor de existência (VE): valor dissociado do uso dos recursos (embora represente consumo ambiental) e derivado de uma posição moral, cultural ou ética em relação aos direitos de existência dos recursos ambientais.

Assim, a valoração ambiental busca estimar as variáveis da VERA:

$$
V E R A=V U D+V U I+V O+V E
$$


Nesse esforço de tentar estimar "preços" para os recursos ambientais e fornecer subsídios técnicos para sua exploração racional, inserem-se os métodos (ou técnicas) de valoração econômica ambiental fundamentados na teoria neoclássica do bem-estar. Os métodos de valoração econômica mais difundidos levam em consideração as preferências individuais (através das quais se obtêm as preferências da sociedade) e a simulação de mercados hipotéticos para a valoração de ativos que não possuem mercados.

A mensuração econômica é um importante instrumento de análise, apesar dos desafios de aplicação, por permitir que um problema com multidimensões seja convertido para uma única unidade. Assim, essa redução permite comparar os diferentes efeitos do projeto (PREST E TURVEY, 1965). A alteração pode ser suficiente para eliminar a confusão criada pelos problemas multidimensionais e fazer com que o processo de escolha seja facilitado.

A análise econômica de projetos e mais especificamente a $\mathrm{ACB}$ tem como base a teoria do bem-estar social pertencente à teoria econômica neoclássica. De acordo com Hanley e Spash (1994), a escolha social e a mensuração do bem-estar consideram uma série de critérios, com destaque para dois: Ótimo de Pareto e o Kaldor-Hicks. O Ótimo de Pareto requer que, ao final da política ou projeto, ninguém se encontre em uma situação pior e que pelo menos uma pessoa esteja em uma situação melhor. No entanto, as políticas normalmente melhoram a situação de algumas pessoas, mas piora a de outras.

Já o Critério Kaldor-Hicks tem como princípio a compensação potencial. Aqueles que usufruem de um benefício podem compensar os que arcaram com os custos. Assim, os benefícios precisam ser superiores aos custos e a compensação é concebível.

A grande limitação do critério Kaldor-Hicks e consequentemente da análise pelo VPL está na incapacidade de avaliar os aspectos distributivos dos impactos avaliados. Em um contexto sustentável, os impactos intergeracionais e intrageracionais precisam ser considerados na adoção de políticas públicas. O problema prático dessa aplicação está na subestimação dos impactos no futuro. Como ao longo do tempo há uma diminuição da representatividade de um valor monetário (cem reais hoje nos trariam maior satisfação do que cem reais em 5 anos), os impactos que foram transformados em unidade monetária também terão seu valor reduzido ao longo do tempo. Implicitamente, o processo de desconto leva a uma maior valorização da geração presente em detrimento das gerações futuras. 
Já na década de 60, Feldstein (1965) afirmava que escolher o tempo de análise dos fluxos de benefícios e custos sociais é um dos problemas mais difíceis e importantes na avaliação de projetos de investimentos públicos. O cálculo da taxa de desconto define uma relação funcional que faz com que as rendas geradas em pontos diferentes no tempo se tornem comensuráveis entre si, atribuindo-lhes valores presentes equivalentes. Determinar a admissibilidade de um projeto requer comparar seu benefício líquido anual com o consumo que teria ocorrido se os recursos não tivessem sido usados no projeto em particular.

O desconto do futuro reflete a preferência pelo presente dos consumidores e a preferência pelo custo de oportunidade do capital pelos produtores (preferência pura pelo tempo). Outra argumentação que justificaria a utilização de uma taxa de desconto positiva está na utilidade marginal decrescente do consumo com o aumento da renda (elasticidade da utilidade marginal) em que quanto maior for a renda das gerações futuras menor será o valor que darão ao aumento marginal do consumo. Consequentemente, quanto maior for a renda das gerações futuras menor será o valor que darão ao aumento marginal do consumo (HANLEY E SPASH, 1994).

Os impactos da definição da taxa de desconto ficam ressaltados em projetos com benefícios ou custos que só serão resgatados no longo prazo. Quanto maior for o horizonte temporal necessário para o custo ou benefício se concretizarem, menor será o valor presente líquido.

No contexto intergeracional, a Fórmula de Ramsey define a taxa de desconto que iguala a soma da taxa de desconto utilitarista e a taxa de crescimento do consumo no período, ponderada pela elasticidade marginal da utilidade do consumo (ARROW et al., 2012):

$$
\rho_{t}=\delta+\eta \cdot g_{t}
$$

em que: $\rho_{t}$ é a taxa de desconto social, $\delta$ é a taxa pura social de preferência temporal (o que pode ser interpretada como a taxa de desconto da utilidade do indivíduo), $\eta$ é a taxa de aversão ao risco relativo e $g_{t}$ é a taxa de crescimento do consumo (elasticidade marginal da utilidade do consumo).

Porém, há uma série de divergências quanto à forma de determinação da taxa de desconto. Hanley e Spash (1994) enumeram as limitações observadas quando se analisa a taxa social de desconto: 
a) A taxa pura social de preferência temporal individual seria diferente da taxa adotada pela sociedade.

A sociedade, de forma coletiva, escolheria poupar mais do que a soma individual das decisões de poupar. Isso ocorre porque poupar agora (e investir agora) gera benefícios de consumo no futuro, o que apresentaria uma oferta inferior ao desejável no mercado por conta do problema dos caronas. Assim, os governos deveriam aplicar uma taxa de desconto inferior à que seria aplicada pelo mercado.

A diferença entre a taxa social de desconto e a individual estaria no fato de que os indivíduos apresentam preferências intertemporais diferentes ao adotarem o papel de cidadão e de consumidor. Provavelmente, os indivíduos adotariam uma taxa de desconto inferior no primeiro papel, especialmente no que tange a custos e benefícios ambientais.

A adoção da taxa de desconto do mercado de longo prazo em análises de políticas públicas é questionada pelo fato de que só é considerada a taxa de preferência pelo tempo da geração corrente. O questionamento está no fato de que os indivíduos apresentam expectativas de vida finitas, o que influencia a taxa, diferentemente da sociedade, que apresenta uma noção coletiva de perpetuidade, reduzindo a taxa pura de preferência temporal.

b) A taxa pura social de preferência temporal individual seria inferior à taxa de retorno do capital.

Outro aspecto que limita a definição da taxa social de desconto está no fato de que ela será inferior à taxa de retorno do capital por conta da taxação. Assim, o setor privado realizaria poucos investimentos do ponto de vista da sociedade.

Além dos aspectos filosóficos, a definição de qual taxa de desconto deve ser adotada em políticas públicas (policies) está sujeita a uma escolha política (politics). Trabalho realizado por Page (1977) analisou o programa de construção de barragens adotado pelos Estados Unidos durante os anos 60 e 70 e observou que caso a taxa de desconto passasse de $2,5 \%$ para $8 \%$, cerca $80 \%$ dos projetos implantados não teriam sido aprovados. De acordo com o trabalho, a estrutura econômica da época permitiria a adoção de uma taxa de desconto de $8 \%$; porém, a escolha prévia da construção das barragens influenciou a análise dos projetos.

Assim, há uma vasta discussão literária acerca da definição da taxa de desconto. As imperfeições e distorções do mercado em relação às premissas teóricas mostram que não há uma taxa única pré-determinada que 
deva ser utilizada. Diversos autores vêm propondo formas de se estimar e aplicar a taxa de desconto sem chegar a um acordo aparente. $O$ trabalho de Saez e Requena (2007) organiza as propostas dos autores de acordo com o método sugerido. Essa classificação foi complementada por trabalhos mais recentes e foi organizada no Quadro 2.

\section{Quadro 2 Resumo dos métodos propostos para a definição da taxa de desconto}

\begin{tabular}{|c|c|}
\hline Proposta e autores & Descrição \\
\hline $\begin{array}{l}\text { Taxa de desconto igual à zero. } \\
\text { Autores: Shue (1999); Harrod } \\
\text { (1948); Ciriary-Wantrup (1942). }\end{array}$ & $\begin{array}{l}\text { Esse seria o único valor adequado para garantir o tratamento } \\
\text { equitativo entre as gerações e discutem o aspecto ético de } \\
\text { se calcular taxas de desconto positivas. Essa abordagem tem } \\
\text { como foco os impactos ecológicos dos projetos em detrimento } \\
\text { das relações econômicas associadas à alocação de recursos } \\
\text { para investimentos. } 0 \text { custo de oportunidade do capital, } \\
\text { o crescimento potencial de consumo e as preferências da } \\
\text { sociedade não são incluídas nesse método. Em termos práti- } \\
\text { cos, seria difícil alocar elevados montantes de recursos para } \\
\text { a adoção de um projeto sem que haja a inclusão dos aspectos } \\
\text { econômicos na análise. }\end{array}$ \\
\hline $\begin{array}{l}\text { Taxa de preferência social } \\
\text { temporal (Social Time } \\
\text { Preference Rate - STPR). } \\
\text { Autores: Roemer (2013); } \\
\text { Dasgupta (2011); Lesser et al. } \\
\text { (1997); Solow (1991). }\end{array}$ & $\begin{array}{l}\text { Acredita-se que uma taxa de desconto positiva está associada } \\
\text { ao acúmulo de capital e tecnologia que será deixado para as } \\
\text { futuras gerações. } 0 \text { cálculo da STPR envolve a premissa de que } \\
\text { as futuras gerações terão uma renda maior e um impacto cau- } \\
\text { sado no futuro seria menor do que o impacto causado pela não } \\
\text { realização do projeto no presente. Além disso, a adoção desse } \\
\text { método parte do pressuposto de que o capital natural pode ser } \\
\text { perfeitamente substituído pelos outros tipos de capital. }\end{array}$ \\
\hline $\begin{array}{l}\text { Inclusão de uma restrição } \\
\text { à redução do capital natural. } \\
\text { Autores: Pearce e Turner (1990); } \\
\text { Barbier et al. (1990). }\end{array}$ & $\begin{array}{l}\text { Não seria necessário reduzir as taxas de desconto se for } \\
\text { incluída na análise uma restrição à redução do capital natural. } \\
\text { Independentemente do impacto, o estoque de capital natural } \\
\text { deve ser constante (compensação ambiental). Contudo, o } \\
\text { ajuste proposto após a inclusão das externalidades no cálculo } \\
\text { dos custos do projeto não seria adequado por levar à dupla } \\
\text { contagem do dano, já que os impactos estariam mensurados } \\
\text { no custo do projeto e na restrição do capital natural. Essa pro- } \\
\text { posta também apresenta uma dificuldade de operacionalização } \\
\text { por conta da definição do limite de redução do estoque natural. }\end{array}$ \\
\hline $\begin{array}{l}\text { Taxa de desconto linearmente } \\
\text { decrescente ao longo do tempo. } \\
\text { Autores: Horta (1998) e } \\
\text { Rabl 1996). }\end{array}$ & $\begin{array}{l}\text { Esse método busca amenizar o viés pelo presente da aplicação } \\
\text { da taxa de desconto com a aplicação linearmente decrescente } \\
\text { da taxa de desconto. Essa adoção é amplamente criticada por } \\
\text { não apresentar critérios claros para a redução da taxa. }\end{array}$ \\
\hline
\end{tabular}

(continua) 
Quadro 2 (continuação)

\begin{tabular}{l} 
Proposta e autores \\
\hline Obtenção da taxa de desconto \\
de forma empírica. \\
Autores: Luckert e Admowicz \\
(1993); Cropper et al. (1994).
\end{tabular}

Descrição

O comportamento e a visão da geração atual seriam mensurados para definir a taxa de desconto. Uma das formas sugeridas é por meio de pesquisa de opinião da geração presente. 0 problema desse método estaria na ausência de análise das perspectivas de mercado e de melhoria de bem-estar (consumo) futuro e as dificuldades na aplicação de pesquisas de opinião para algo não associado de forma concreta à vida da população atual (viés positivo de preocupação com a geração futura).

Adoção da taxa de desconto
padrão com aumento do valor
do meio ambiente ao longo
do tempo.
Autores: Porter (1982);
Krutilla e Fisher (1975).

Explicitação das gerações futuras na análise. Autores: Chichilnisky (2009); Sumaila e Walters (2005).
Essa proposta prevê que a taxa de desconto seja estimada com base no mercado financeiro e que o valor mensurado dos impactos ambientais aumente ao longo do tempo. A dificuldade da aplicação desse método está na falta de clareza da taxa de crescimento do impacto ambiental. A proposta ficaria sujeita à visão do gestor público sobre a relevância daquele impacto, o que poderia gerar um viés político (politics) no processo decisório.

A explicitação das gerações futuras na análise pode ser feita de diversas formas: por meio do cálculo do valor presente líquido ajustado com a incorporação de uma variável intergeracional no modelo (como um nível individual atual de "altruísmo") ou pela inclusão de um fator de desconto entre gerações, entre outras. 0 método apresenta propostas e resultados diferentes dependendo do autor. Ainda não há potencial de discussão para um método de aplicação plausível.

0 método sugere a aplicação de um critério utilitarista hierarquizado de desconto na busca da equidade intergeracional. Os autores propõem que o gestor público utilize um elemento da classe da função de bem-estar social utilitarista hierarquizada, ou seja, com a construção de uma função de bem-estar social em um horizonte temporal infinito onde a utilidade da geração seguinte deve ser igual ou superior à utilidade da geração anterior. A proposta não demonstra com clareza como o método poderia ser aplicado. Alguns desenvolvimentos foram realizados no caso de mudanças climáticas, mas sem aplicação clara em políticas públicas.

Taxa de desconto dual. Autores: Kula e Evans (2011); Saez e Requena (2007).
Os autores propõem a aplicação de uma taxa de desconto para os impactos tangíveis e outra para os intangíveis (ambientais). Contudo, o trabalho elaborado por Arrow et al. (2012) para a construção de um método de definição da taxa de desconto desaconselha a aplicação de taxas de desconto diferentes para um mesmo período no tempo. Essa diferença geraria inconsistências intrageracionais e intergeracionais. 
Quadro 2 (continuação)

\begin{tabular}{l|l}
\hline Proposta e autores & Descrição \\
\hline Taxa de desconto baseada no & A taxa de desconto adotada sofreria variações de acordo com o \\
comportamento mercado. & comportamento atual do mercado perante fatores como risco, \\
Autores: Arrow et al. (2012), & propensão a poupar, consumo, entre outros. Em muitos casos, \\
Mertens e Rubinchik (2011). & $\begin{array}{l}\text { os autores sugerem a adoção de uma taxa decrescente de } \\
\text { desconto em formato de hipérbole. Como: Gowdy et al. (2013), } \\
\end{array}$ \\
\hline
\end{tabular}

Fonte: Elaboração própria com base nas referências mencionadas.

Portanto, uma série de discussões foi desencadeada a respeito da importância e definição da taxa de desconto na análise de projetos. $O$ método proposto por Arrow et al. (2012) afirma que a taxa decrescente de desconto deve ser usada quando há incertezas relacionadas ao estado da economia e quando também há choques de crescimento persistentes. Nesse caso, duas abordagens poderiam ser adotadas. A primeira seria estruturar modelos estocásticos de estimação da taxa de juros de mercado. A segunda seria utilizar a versão estendida da Fórmula de Ramsey em que $\delta$ e $\eta$ seriam definidos e uma modelagem seria desenvolvida para definir $g_{t}$ conforme exposto na Equação 2 (citada anteriormente):

$$
\rho_{t}=\delta+\eta \cdot g_{t}
$$

A parametrização da Fórmula de Ramsey também é discutida com base em duas abordagens: descritiva e normativa. A abordagem descritiva pressupõe que a taxa de desconto deva refletir o comportamento observado em mercados. A normativa requer que aspectos éticos sejam levados em consideração na estimativa da taxa de desconto da utilidade do indivíduo $(\delta)$ e a elasticidade marginal da utilidade do consumo $g_{t}$. De forma relativamente consensual, sugere-se que:

$\delta$ seja próximo de zero por representar a utilidade das futuras gerações. Nesse sentido, seria aceitável apenas um valor que represente a probabilidade das futuras gerações não existirem.

$\eta$ determine a velocidade da queda da utilidade marginal do consumo na medida em que o consumo aumenta. Em outras palavras, reflete o sacrifício máximo que uma geração estaria disposta a transferir de renda para outra geração. Esse parâmetro também mede o coeficiente de aversão relativa ao risco e a elasticidade intertemporal da substituição de consumo. 
Uma forma de estimar $\eta$ seria observando as políticas de transferência de renda utilizadas na sociedade, como é o caso da tributação progressiva de renda, por exemplo. Seria possível afirmar que a aversão à inequidade intrageracional seria similar à intergeracional. Quanto menor o valor, maior a propensão da sociedade a poupar para aumentar o bem-estar de futuras gerações. Há também uma visão em que $\eta$ representaria o coeficiente de aversão ao risco, o que poderia ser observado no mercado financeiro. O problema é que essa abordagem, por ser restrita aos agentes ativos no mercado financeiro, não reflete os trade-offs intrageracionais de consumo, o que limita a análise de bem-estar social.

Já $g_{t}$ seria a taxa de crescimento do consumo.

Porém, como há uma série de incertezas relacionadas à taxa de crescimento de consumo, Arrow et al. (2012) sugerem uma extensão da Fórmula de Ramsey com taxa de crescimento de consumo incerta:

$$
\rho=\delta+\eta \mu_{g}-0,5 \eta^{2} \sigma_{g}^{2}
$$

em que a variação proporcional no consumo no momento $i$ normalmente distribuído com média $\mu_{g}$ e variância $\sigma_{g}^{2}$. Os últimos termos da fórmula apresentam um efeito de precaução onde a incerteza sobre a taxa de crescimento do consumo reduz a taxa de desconto, fazendo com que o gestor público poupe mais no presente. Esse fator, contudo, deverá apresentar baixo valor.

Outros impactos, como grandes choques no consumo oriundos de uma grande crise econômica ou guerras, apresentam impactos significativos na taxa de desconto. Nesse caso, supõe-se que $x_{t}$ represente a taxa de crescimento do consumo em porcentagem no ano $t$. $O$ crescimento no consumo apresentaria distribuição normal com média $\mu_{g}$ e variância $\sigma_{g}^{2}$. A variável $\mu_{g}$ é estimada com base em n observações sobre $x_{t}$. Isto conduz à seguinte equação para a taxa de desconto de segurança-equivalente:

$$
\rho=\delta+\eta \mu_{g}-0,5 \eta^{2} \sigma_{g}^{2}-0,5 \eta^{2} \sigma_{g}^{2}(t / n)
$$

O quarto termo presente na equação, de acordo com os autores, tem um "efeito de previsão estatística" que faz $\rho$ diminuir na medida em que t aumenta condicionada à variância $\left(\sigma_{g}^{2}\right)$. Em outras palavras, a incerteza sobre a taxa média de crescimento do consumo influencia a tendência decrescente da taxa de desconto. 
O trabalho de Arrow et al. (2012) conclui que, na Fórmula de Ramsey, a incerteza sobre a taxa de crescimento per capita de consumo pode levar a uma taxa de desconto decrescente de consumo ao assumir que os choques de consumo estão positivamente relacionados. Essa incerteza da taxa de crescimento futuro pode ser estimada econometricamente, baseando-se em observações históricas, ou pode ser derivada da incerteza subjetiva relacionada à taxa de crescimento médio no consumo médio.

O Valor Presente Líquido Esperado (VPLE) é considerado teoricamente menos elegante, além de não medir a taxa de desconto do consumo, o que pode ser obtido na Fórmula de Ramsey. Porém, o VPLE é empiricamente factível.

Outra sugestão relevante feita por Arrow et al. (2012) é a ressalva de que não se deve usar taxas de desconto diferentes em um mesmo ano para fatores diferentes. $O$ mais adequado seria adotar uma taxa de desconto decrescente, resultando em uma consistência mais adequada entre os aspectos inter e intrageracionais. Ou ainda pode-se adotar a mesma taxa de desconto constante e exponencial para todas as categorias de custo de benefício.

\section{Equidade intergeracional e a taxa de desconto para o caso do setor elétrico brasileiro: um exemplo}

A escolha de uma matriz energética envolve também a escolha de quais impactos ambientais serão considerados menos nocivos pela sociedade. O processo político do planejamento da matriz energética deveria idealmente refletir as escolhas da sociedade que representa, mas para que a decisão seja tomada é importante que todas as possibilidades de custos e benefícios das fontes de energia disponíveis sejam consideradas e tornadas claras e disponíveis.

No entanto, algumas fontes de geração de energia elétrica envolvem uma série de incertezas quanto aos custos potenciais, como é o caso da geração nuclear e da construção de usinas hidrelétricas com grandes reservatórios. A limitação das informações afeta os instrumentos técnicos que deveriam ser utilizados no processo de decisão.

Para ilustrar a questão, um exemplo aqui discutido é o da Usina Nuclear de Angra 3. Nesse caso, fica clara a falta de detalhamento de impactos no Estudo de Impacto Ambiental e no Relatório de Impacto Ambiental - EIA/ 
RIMA (ELETRONUCLEAR, 2005). Os longos documentos descrevem os biomas da área potencialmente afetada, mas não descrevem como eles seriam afetados nem tampouco quais seriam as consequências. Sabe-se que as incertezas quanto aos impactos potenciais no longo prazo são muitas; contudo, no EIA/RIMA não há qualquer exercício ou tentativa de estimativa de como a área seria afetada no curto prazo e muito menos no longo prazo. Pode-se inclusive afirmar que tais custos seriam considerados invisíveis, já que não são analisados ou mencionados.

A falta de análise desses "custos invisíveis" está relacionada aos limites científicos da mensuração dos impactos ambientais, como é o caso de se mensurar aquilo que é incerto ou muito distante temporalmente dos tomadores de decisão. As limitações científicas e incertezas relacionadas aos impactos no longo prazo geram também incertezas quanto à mensuração dos parâmetros avaliados. Essa falta de informação faz com que a análise subestime ou ignore tais impactos sem que haja uma consideração qualitativa.

Contudo, a ausência de informações deveria reforçar a cautela no processo de decisão de empreendimentos como esse e a mensuração de tais impactos, mesmo com suas limitações, ainda é primordial.

De acordo com a Agência Nacional de Energia Elétrica (2014), a taxa de desconto do setor é de $12 \%$ ao ano. Porém, ao considerar o aspecto intergeracional, a taxa de desconto adequada para projetos do setor elétrico com impactos no longo prazo poderia ser mensurada pela Fórmula de Ramsey estendida de segurança-equivalente:

$$
\rho=\delta+\eta \mu_{g}-0,5 \eta^{2} \sigma_{g}^{2}-0,5 \eta^{2} \sigma_{g}^{2}(t / n)
$$

onde:

$\rho$ : taxa de desconto social.

$\delta$ : probabilidade das futuras gerações não existirem.

$\eta$ : aversão à inequidade intertemporal.

$\mu_{g}:$ média da taxa de crescimento do consumo.

$\sigma_{g}^{2}$ : variância da taxa de crescimento do consumo.

$t$ : período analisado

$n$ : tamanho da amostra

A variável $\delta$ foi estimada por Stern (2006) como $0,1 \%$ ao ano. 
Em nosso exemplo, a definição de $\eta$ terá como base o trabalho de Tol (2010), que estimou a aversão à desigualdade internacional no consumo como expressa na ajuda ao desenvolvimento dada pelos países ricos aos países pobres entre 1965 e 2005. O valor encontrado foi de 0,7.

Para calcular a média e o desvio padrão da taxa de crescimento do consumo, iremos considerar a variação real das famílias entre 1993 e 2013, conforme apresentado na Tabela 1.

Tabela 1 Resumo estatístico da estimativa da taxa de crescimento do consumo das famílias

\begin{tabular}{lr}
\hline Variável & Valor \\
\hline Tamanho da amostra $(\mathrm{n})$ & 21 \\
\hline Média amostral $(\overline{\mathrm{X}})$ & 3,7418 \\
\hline Desvio padrão da amostra $(\mathrm{S})$ & 2,5341 \\
\hline Variância da amostra $\left(\mathrm{S}^{2}\right)$ & 6,4216 \\
\hline
\end{tabular}

Fonte: Elaboração própria, com base em IPEADATA(2014).

Com base na Tabela 2 e nos demais parâmetros citados, a Fórmula de Ramsey estendida de segurança-equivalente pode ser calculada como:

$$
\begin{aligned}
& \rho=0,1+0,7 \times 3,7418-0,5 \times 0,7^{2} \times 6,4216-0,5 \times 0,7^{2} \times 6,4216(t / 21) \\
& \rho=1,1460-1,5733(t / 21)
\end{aligned}
$$

O Gráfico 1 apresenta a evolução da taxa de desconto para o exemplo. Observa-se que o valor inicial da taxa é de 1,07 e se torna negativo a partir do ano 2033. A diferença entre as duas taxas de desconto é significativa.

A elevada taxa de crescimento de consumo das famílias no Brasil, assim como o elevado desvio padrão apresentam um efeito de precaução onde a incerteza sobre a taxa de crescimento do consumo reduz a taxa de desconto, fazendo com que o gestor público poupe mais no presente. Em outras palavras, a taxa de crescimento do consumo das famílias brasileiras apresenta um comportamento volátil, o que aumenta a precaução, já que o impacto negativo pode ser elevado no futuro. Essas variáveis são fundamentais para que os custos no longo prazo sejam considerados no presente.

O desenvolvimento de um exercício simples deixa claro essa diferença. Suponha um projeto de energia elétrica X que apresente um custo ambiental 
anual constante no valor de $\mathrm{R} \$ 1.000,00$ a título de simplificação matemática e um horizonte temporal de 100 anos. Vale destacar que, no caso de depósito de resíduos nucleares, a International Atomic Energy Agency (IAEA, 2002) recomenda que os depósitos finais de resíduos nucleares precisam de acompanhamento ativo também no período após os encerramentos das atividades e sugere que esse acompanhamento institucional seja realizado durante 300 anos, período equivalente a 10 meias-vidas de alguns importantes rejeitos de médias e baixas atividades como o Césio e o Estrôncio. Ou seja, o horizonte temporal de 100 anos seria bem inferior ao período ideal de análise.

\section{Gráfico 1 Evolução da taxa de desconto calculada pela Fórmula de Ramsey estendida de segurança-equivalente}

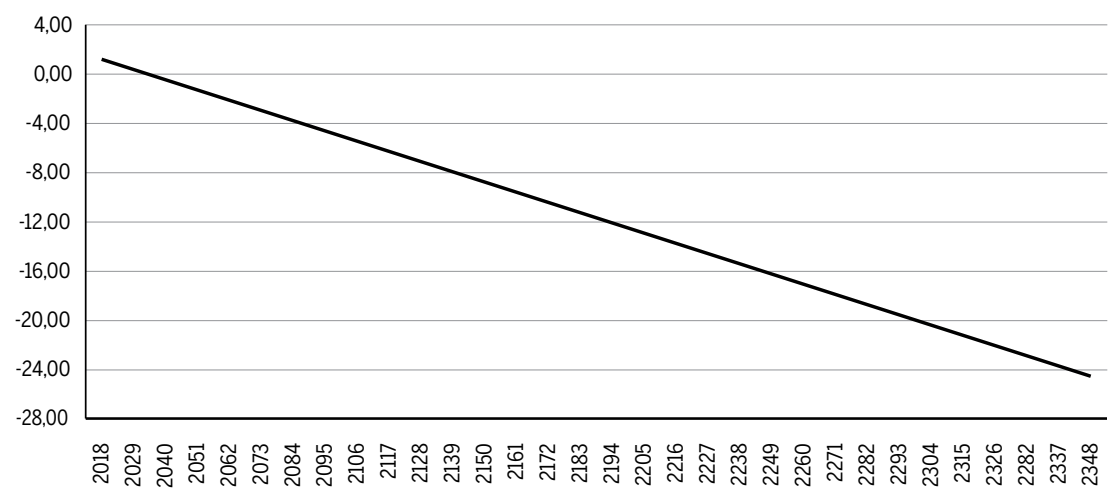

Fonte: Elaboração própria.

\section{Gráfico 2 Comparação das curvas anuais do valor presente}

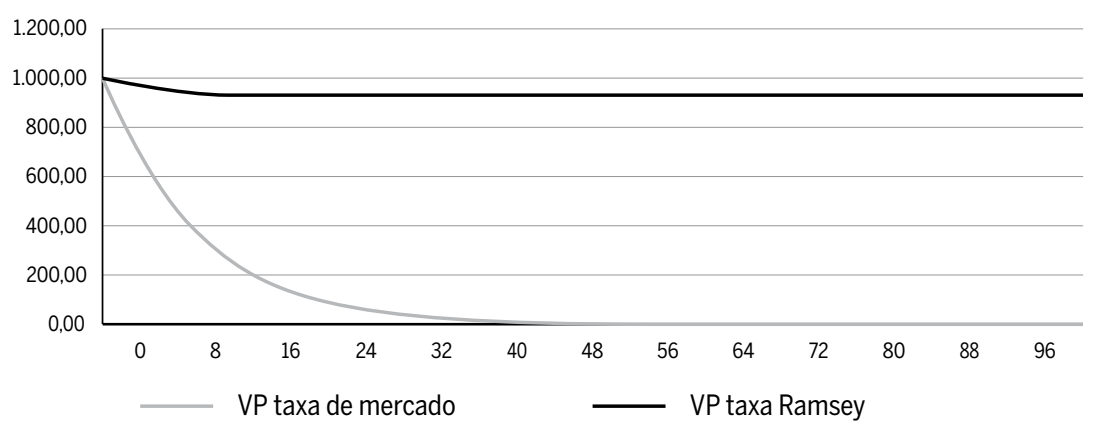

Fonte: Elaboração própria. 
Ao aplicarmos a taxa de desconto do mercado de $12 \%$ ao ano, o Valor Presente total desse custo ambiental seria de $R \$ 9.333,23$. Já com a aplicação da taxa de desconto baseada na Fórmula de Ramsey estendida de segurança-equivalente, o Valor Presente total seria de $\mathrm{R} \$ 94.419,87$, dez vezes superior ao valor estimado com a taxa de desconto de mercado (Gráfico 2).

Essa diferença pode ser ainda maior dependendo do nível de aversão à inequidade da sociedade representada no modelo. $O$ trabalho de Tol (2010) estima que, para qualquer padrão de doação e qualquer aversão ao risco, o nível de aversão a inequidade parte do pressuposto de que o fluxo de doações é ótimo ou seja que a derivada do bem-estar social é igual a zero da seguinte fórmula:

$$
\sum_{\text {Doadores }} p_{D} c_{D}^{\eta \varpi-\eta-\sigma} a+\sum_{\text {Receptores }} p_{R} c_{R}^{\eta \varpi-\eta-\sigma} a=0
$$

onde:

$p_{D}:$ população que realiza doações

$c_{D}$ : consumo médio per capita mensal da população que realiza doações

$a$ : doação per capita recebida

$p_{R}$ : população que recebe as doações

$c_{R}$ : consumo médio per capita mensal da população que recebe doações

$\eta$ : aversão à inequidade

$\varpi$ : aversão ao risco

No caso do Brasil, foram consideradas as informações do Instituto para o Desenvolvimento do Investimento Social - IDIS (2016) sobre o perfil dos brasileiros que fazem doações. A Pesquisa Doação Brasil mostra uma série de características dos doadores brasileiros, como renda, frequência e montante doado em 2015. Enquanto o trabalho de Tol (2010) focou na doação entre países, a análise aqui apresentada irá focar na doação entre as diferentes classes sociais. Por conta da indisponibilidade de dados, não é possível analisar a aversão à inequidade do brasileiro ao longo do tempo, mas somente para 2015. A Tabela 2 apresenta um resumo dessas informações. A aversão ao risco adotada para o Brasil foi a calculada por Issler e Siqueira (2000) no valor de 4,89. 
Tabela 2 Informações estatísticas sobre o perfil dos doadores brasileiros em 2015

\begin{tabular}{lr}
\hline Variável & Valor \\
\hline Número de doadores & 31.346 .540 \\
\hline Número de beneficiados $^{1}$ & 81.189 .684 \\
\hline Consumo per capita mensal dos doadores $^{2}$ & $\mathrm{R} \$ 2.213,38$ \\
\hline Consumo per capita mensal dos beneficiados $^{3}$ & $\mathrm{R} \$ 161,24$ \\
\hline Doação per capita mensal realizada $^{\text {Doação per capita mensal recebida4 }}$ & $\mathrm{R} \$ 20,00$ \\
\hline
\end{tabular}

Fonte: Elaboração própria, com base em IDIS (2016), IBGE (2016), Neves (2005).

1 População sem renda ou com até um salário mínimo conforme informações do PNAD (2015).

${ }^{2} e^{3}$ Calculado com base na renda mensal per capita média por faixa salarial e propensão marginal a consumir $(0,76)$ do brasileiro (NEVES, 2005).

${ }^{4}$ Representa o volume total doado dividido pelo total de beneficiários.

Considere que:

$$
X=\eta \varpi-\eta-\varpi
$$

Logo:

$$
p_{D} c_{D}^{X}(-a)+p_{R} c_{R}^{X} a=0
$$

Adotando as variáveis apresentadas na Tabela 1, temos:

$$
\begin{aligned}
& 31.346 .540 \times 2.213,38^{X} \times(-) 20+81.189 .684 \times 161,24^{X} \times 7,72=0 \\
& \log 1=(X \times \log 161,24-X \times \log 2.213,38) \\
& 0=-1,1376 \times X
\end{aligned}
$$

Substituindo (7) em (8):

$$
\begin{aligned}
& 0=-1,1376 \times(4,89 \times \eta-4,89-\eta) \\
& \eta=1,2571
\end{aligned}
$$

Ou seja, para o ano de 2015, a taxa de aversão à inequidade do brasileiro 
seria de 1,2571. Em termos práticos, a tendência da taxa de desconto seria em um valor ainda inferior.

Com base na análise de Padilla (2002) e na diferença observada nas diferentes taxas de desconto, considera-se relevante que projetos com impactos ambientais irreversíveis no longo prazo incluam em seus cálculos o custo de tais impactos com o uso de uma taxa de desconto apropriada. Assim, a análise de viabilidade do projeto ou sua análise custo-benefício internalizará os custos que serão arcados pelas gerações no longo prazo.

\section{Comentários finais}

A mensuração econômica converte vários impactos a uma única unidade; a taxa de desconto também exerce esse papel ao converter uma unidade monetária com diferentes valores (como poder de compra e custo de oportunidade) em uma única unidade monetária ao longo do tempo. Essa conversão ao longo do tempo pode reduzir a zero os impactos potenciais com custo não nulo. Nesse sentido, é importante considerar o método proposto por Arrow et al. (2012), em que a taxa decrescente de desconto deve ser usada quando há incertezas relacionadas ao estado da economia e quando também há choques de crescimento persistentes, contexto coerente com análises de longo prazo. A Fórmula de Ramsey seria a indicada para esses casos.

Para o caso do setor elétrico brasileiro, a diferença entre a taxa de desconto de mercado e a calculada na Fórmula de Ramsey é discrepante especialmente pela grande variação no consumo de famílias e pelo elevado nível de aversão à inequidade dos brasileiros. Esse resultado mostra um grande distanciamento entre os cálculos tradicionais de desconto e o que é refletido na sociedade brasileira.

Com base na análise de Padilla (2002) e na diferença observada na mensuração do custo ambiental do exemplo estudado quando diferentes taxas de desconto são aplicadas, considera-se relevante que projetos com impactos ambientais irreversíveis no longo prazo incluam em seus cálculos o custo de tais impactos com o uso de uma taxa de desconto apropriada. Recomenda-se o uso da Fórmula de Ramsey de Segurança Equivalente pela disponibilidade de informações e por considerar eventos possíveis no longo prazo, como choques no consumo oriundos de uma grande crise 
econômica ou guerras. Assim, a análise de viabilidade do projeto ou sua análise custo-benefício internalizará os custos que serão arcados pelas gerações no longo prazo.

Nesse contexto, e com base no trabalho de Padilla (2002), sugere-se aliar a adoção da precaução a formas de controle para acompanhar se um dano irreversível irá ocorrer. Se a interferência prevista no projeto pode ser evitada, principalmente quando se trata de um projeto rentável, essa postura deve ser adotada ou ainda implementar projetos complementares com o intuito compensatório. Essa alternativa envolve uma avaliação prévia da eficácia do projeto no que tange à compensação do dano causado. Além disso, é urgente que haja uma análise interinstitucional (ministérios, agências reguladoras, e etc) sobre tais aspectos de modo a internalizar a equidade intergeracional no processo decisório e refletir os indicadores observador na sociedade brasileira.

\section{Referências}

AGÊNCIA NACIONAL DE ENERGIA ELÉTRICA. Avaliação das contribuições da AP 086/2013, que tratou de obter subsidios para a homologação dos programas computacionais de planejamento e formação de preço com internalização de mecanismos de aversão a risco de que trata o art. $1^{\circ}$ da Resolução CNPE no 03/2013. Nota Técnica nº 059/2013. 26 p., 2013.

ARROW, K. Discounting, morality, and gaming. In: PORTNEY, P. WEYANT, J. Discounting and Intergenerational Equity. Washington: Resources for the future, p. 13-21, 1999.

ARROW, K.; CROPPER, M.; GOLLIER, C.; GROOM, B.; HEAL, G.; NEWELL, R.; NORDHAUS, W.; PINDYCK, R.; PIZER, W.; PORTNEY, P.; STERNER, T.; TOL, R.; WEITZMAN, M. How Should Benefits and Costs Be Discounted in an Intergenerational Context? Washington: Resources for the future, 34 p., 2012.

ASHEIM, G.; BUCHHOLZ, W; TUNGODDEN, B. Justifying Sustainability. Journal of Environmental Economics and Management, v. 41, p. 252-268, 2001.

ASSOCIAÇÃO BRASILEIRA DE NORMAS TÉCNICAS - ABNT. Avaliação de bens Parte 6: Recursos naturais e ambientais. Norma ABNT NBR Brasileira 14653-6, 21 p., 2008.

BANCO CENTRAL DO BRASIL. Relatório de Inflação, v. 16, n. 1, 115 p., 2014.

BANCO CENTRAL DO BRASIL. Taxas de câmbio. Disponível em: <http://www.bcb.gov.br/ ?txcambio>. Acesso em: 17 abr. 2014.

BARBIER, E.; MARKANDYA, A.; PEARCE, D. Environmental sustainability and Cost-Benefit Analysis. Environment and Planning, v. 22, p. 1259-1266, 1990.

BERGH, J. Externality or sustainability economics? Ecological Economics, v. 69, p. 2047-2052, 2010. BRUNDTLAND, G. Our Common Future. The World Commission on Environment and Develop- 
ment. Oxford University: Oxford University Press, p. 37, 1987.

CHICHILNISKY, G. Avoiding extinction: equal treatment of the present and the future. Economics: The Open-Access, Open-Assessment E-Journal, v. 3, 32 p., 2009.

CHICHILNISKY, G. Sustainable markets with short sales. Economic Theory, v. 49, p. 293-307, 2012.

CIRIACY-WANTRUP, S. Private enterprise and conservation. Journal of Farm Economy, v. 24, p. 75-96, 1942.

CONTADOR, C. Cálculo dos benefícios e custos sociais. In: Projetos sociais: avaliação e prática. 4. ed. São Paulo: Atlas, p. 78-10, 2000.

CROPPER, M.; AYDADE, S.; PORTNER, P. Preferences for livesaving programs: how the public discount time and age. Journal of Risk and Uncertainty, v. 8, p. 243-246, 1994.

DASGUPTA, P. The Ethics of Intergenerational Distribution: Reply and Response to John E. Roemer. Environmental and Resource Economics, v. 50, p. 475-493, 2011.

DASGUPTA, P.; HEAL, G. The Optimal Depletion of Exhaustible Resources. Review of Economic Studies, Symposium on the Economics of Exhaustible Resources, v. 41, p. 3-28, 1974.

DASGUPTA, S.; MITRA, T. Intergenerational Equity and Efficient Allocation of Exhaustible Resources. International Economic Review, v. 24, p. 133-153, 1983.

ELETRONUCLEAR. Estudo de Impacto Ambiental da Unidade 3 da Central Nuclear Almirante Álvaro Alberto - ANGRA 3. 2162 p., 2005.

FELDSTEIN, M. The Social Time Preference Discount Rate in Cost Benefit Analysis. The Economic Journal, v. 74, p. 360-379, 1965.

FREDERICK, S.; LOEWENSTEIN, G.; O'DONOGHUE, T. Time discounting and time preference: a critical review. Journal of Economics Literature, v. 40, p. 351-401, 2002.

FUNDAÇÃO GETULIO VARGAS. Índice Geral de Preços - Mercado (IGP-M). Disponível em: < http://www.ipeadata.gov.br/>. Acesso em: 17 abr. 2014.

GOWDY, J.; ROSSER JR, J.; ROY, L. The evolution of hyperbolic discounting: Implications for truly social valuation of the future. Journal of Economic Behavior \& Organization, v. 905, p. 594-610, 2013.

HANLEY, N.; SPASH, C. Cost-Benefit Analysis and the Environment. Hampshire: Edward Elgar Publishing Limited, p. 27-151, 1994.

HARROD, R. Towards a Dynamic Economy. London: St. Martin ‘s Press, 169 p., 1948.

HORTA, M. Sobre el interés general de Itoiz y el Canal de Navarra. Ingeniería del Agua 5, v. 2, p. 63-74, 1998.

HOWARTH, R.; NORGAARD, R. Environmental Valuation under Sustainable Development. American Economic Review, v. 82, p. 473-77, 1992.

HOWARTH, R.; NORGAARD, R. Intergenerational Resource Rights, Efficiency, and Social Optimality. Land Economics, v. 66, p. 1-11, 1990.

INSTITUTO PARA O DESENVOLVIMENTO DO INVESTIMENTO SOCIAL - IDIS. Pesquisa Doação Brasil, 84 p., 2016. Disponível em: <http://idis.org.br/pesquisa-doacao-brasil/>. Acesso em: 10 fev. 2017. 
INTERNATIONAL ATOMIC ENERGY AGENCY. Socio-economic and other non-radiological impacts of the near surface disposal of radioactive waste. Áustria: Vienna, 32 p., 2002.

IPEADATA. Consumo final das famílias: variação real anual. Disponível em: <http://www.ipeadata.gov.br/>. Acesso em: 17 abr. 2014.

ISSLER, J.; PIOUEIRA, N. Estimando a Aversão ao Risco, a Taxa de Desconto Intertemporal, e a Substutibilidade Intertemporal do Consumo no Brasil usando Três tipos de Função Utilidade. Ensaios Econômicos - FGV, n. 387, 2000.

KRUTILLA, J.; FISHER, A. The Economics of Natural Environments. Baltimore: Johns Hopkins University Press, 292 p., 1975.

KULA, E.; EVANS, D. Dual discounting in cost-benefit analysis for environmental impacts. Environmental impact assessment review, v. 31, p. 180-186; 2011.

LESSER, J.; DODDS, D.; ZERBE, R. Environmental Economics and Policy. Addison Wesley Longman, 751 p., 1997.

LUCKERT, M.; ADMOWICZ, W. Empirical measures of factors affecting social rates of discount. Environmental and Resource Economics, v. 3, p. 1-21, 1993.

LUTZ, E.; MUNASINGHE, M. Integration of environmental concerns into economic analyses of projects and policies in an operational context. Ecological Economics, v. 10, p. 37-46, 1994.

MERTENS, J.; RUBINCHIK, A. Intergenerational equity and the discount rate for policy analysis. Macroeconomic Dynamics, p. 1-33, 2011.

MEZ, L. Nuclear energy-Any solution for sustainability and climate protection? Energy Policy, v. 48, p. $56-63,2012$.

MUELLER, C. A economia da sobrevivência: fundamentos e visão analítica. In: Os economistas e as relações entre o sistema econômico e o meio ambiente. Brasília: Finatec, p. 134-146, 2007.

NEVES, E.; CROCOMO, F. A relação entre a pobreza e o crescimento econômico do Brasil: uma análise via a propensão marginal a consumir. 2005. Disponível em: <www.unimep.br/phpg/ mostraacademica/anais/4mostra/pdfs/546.pdf>. Acesso em: 15 fev. 2017.

OKUN, A. Equity and efficiency: the big trade-off. Brookings Institution Press, 124 p, 1975.

PADILLA, E. Intergenerational equity and sustainability. Ecological Economics, v. 41, p. 69-83, 2002.

PAGE, T. Conservation and economic efficiency. Baltimore: John Hopkins University, 266 p., 1977.

PAGE, T. On the Problem of Achieving Efficiency and Equity, Intergenerationally. Land Economics, v. 73, p. 580-96, 1997.

PASCUAL, U.; MURADIAN, R.; RODRÍGUEZ, L.; DURAIAPPAH, A. Exploring the links between equity and efficiency in payments for environmental services: A conceptual approach. Ecological Economics, v. 69, p. 1237-1244, 2010.

PEARCE, D., TURNER, R. Economics of Natural Resources and the Environment. Baltimore: The Johns Hopkins University Press, Harvester Weats Leaf, Hertfordshire, 392 p., 1990.

PORTER, R. The new approach to wilderness preservation through Benefit-Cost Analysis. Journal of Environmental Economics and Management, v. 9, p. 63-80, 1982. 
PREST, A.; TURVEY, R. Cost-Benefit Analysis: A Survey. Economic Journal, v. 75, p. 683-735, 1965.

RABL, A. Discounting of long term cost: what would future generations prefer us to do? Ecological Economics, v. 17, p. 137-145, 1996.

RAWLS, J. A Theory of justice. Harvard University Press, 538 p., 1971.

REVESZ, R.; LIVERMORE, M. Retaking Rationality: How cost-benefit analysis can better protect the environment and our health. New York: Oxford University Press, 254 p., 2008.

ROEMER, J. Once more on intergenerational discounting in climate-change analysis: reply to Partha Dasgupta. Environmental Resource Economics, v. 56, p. 141-148, 2013.

ROEMER, J. The ethics of intertemporal distribution in a warming Planet. Environmental Resource Economics, v. 48, p. 363-390, 2011.

SAEZ, C.; REQUENA, J. Reconciling sustainability and discounting in cost-benefit analysis: a methodological proposal. Ecological Economics, v. 60, p. 712-725, 2007.

SEROA DA MOTTA, R. Manual para Valoração Econômica de Recursos Ambientais. Brasília: Ministério do Meio Ambiente, 254 p., 1997.

SHUE, H. Bequeathing hazards: security rights and property rights of future humans. In: DORE, M.; MOUNT, T. Global Environmental Economics: Equity and the Limits to Markets. Oxford: Willey-Blackwell, 355 p., 1999.

SOLOW, R. Intergenerational equity and exhaustible resources. Review of Economic Studies, v. 41, p. 29-45, 1974.

SOLOW, R. Sustainability: an economists perspective. In: DORFMAN, R.; DORFMAN, N. Economics of the Environment. New York: W.W Norton and Co, 426 p., 1991.

STERN, N. Review on the economics of climate change. London: HM Treasury, 662 p., 2006.

STIGLITZ, J. Growth with Exhaustible Natural Resources: Efficient and Optimal Growth Paths. Review of Economic Studies, Symposium on the Economics of Exhaustible Resource, v. 41, p. 123137, 1974.

SUMAILA, U.; WALTERS, C. Intergenerational discounting: a new intuitive approach. Ecological Economics, v. 52, p. 135-142, 2005.

THUROW, L. Equity, Efficiency, Social Justice, and Redistribution. Nebraska Journal of Economics and Business, v. 20, p. 5-24, 1981.

TOL, R. International Inequity Aversion and the Social Cost of Carbon. Climate Change Economics, v. 1, p. 21-32, 2010.

UNITED KINGDON ENERGY RESEARCH CENTER. The UK energy system in 2050: Comparing Low-Carbon, Resilient Scenarios. London: UKERC Publications Catalogue, 68 p., 2013.

ZUBER, S.; ASHEIM, G. Justifying social discounting: The rank-discounted utilitarian approach. Journal of Economic Theory, v. 147, p. 1572-1601, 2012.

\section{Sobre as autoras}

Ilana Dalva Ferreira - ilana.eco@gmail.com

Confederação Nacional da Indústria - CNI, São Paulo, São Paulo, Brasil.

ORCID: https://orcid.org/0000-0002-7732-7090. 
Ferreira \& Imbroisi

DeniseImbroisi -imbroisi@unb.br

Universidade de Brasília - UnB, Brasília, DF, Brasil.

ORCID: https://orcid.org/0000-0001-5747-5806.

\section{Sobre o artigo}

Recebido em 05 de maio de 2015. Aprovado em 02 de maio de 2017. 